\title{
Craniofacial Asymmetry in Adults With Neglected Congenital Muscular Torticollis
}

\author{
Kil-Yong Jeong, MD, Kyung-Jay Min, MD, Jieun Woo, MD, Shin-Young Yim, MD, PhD
}

The Clinic for Torticollis, Department of Physical Medicine and Rehabilitation, Ajou University School of Medicine, Suwon, Korea

Objective To evaluate the craniofacial asymmetry in adults with neglected congenital muscular torticollis (CMT) by quantitative assessment based on craniofacial three-dimensional computed tomography (3D-CT).

Methods Preoperative craniofacial asymmetry was measured by 3D-CT for 31 CMT subjects $\geq 18$ years of age who visited a tertiary medical center and underwent 3D-CT between January 2009 and December 2013. The relationship between the age and the severity of craniofacial asymmetry was analyzed in reference to anteroposterior length asymmetry of the frontal bone and zygomatic arch, vertical and lateral displacements of the facial landmarks, and mandibular axis rotation.

Results The age at CT was 27.71 \pm 7.02 years (range, 18-44 years). All intra-class correlation coefficients were higher than 0.7 , suggesting good inter-rater reliability $(\mathrm{p}<0.05)$ of all the measurements. The frontal and the zygomatic length ratio (i.e., the anteroposterior length asymmetry on the axial plane) was $1.06 \pm 0.03$ and $1.07 \pm 0.03$, respectively, which was increased significantly with age in the linear regression analysis $\left(\mathrm{r}^{2}=0.176, \mathrm{p}=0.019\right.$ and $r^{2}=0.188, p=0.015$, respectively). The vertical or lateral displacement of the facial landmarks and rotation of the mandibular axis did not significantly correlate with age ( $p>0.05)$.

Conclusion Craniofacial asymmetry of neglected CMT became more severe with age in terms of anteroposterior length asymmetry of the ipsilateral frontal bone and zygomatic arch on the axial plane even after growth cessation. This finding may enhance the understanding of therapeutic strategies for craniofacial asymmetry in adults with neglected CMT.

Keywords Craniofacial abnormalities, Facial asymmetry, Computed tomography, Congenital muscular torticollis

Received July 30, 2014; Accepted October 16, 2014

Corresponding author: Shin-Young Yim

The Clinic for Torticollis, Department of Physical Medicine and Rehabilitation, Ajou University School of Medicine, World cup-ro 164, Yeongtong-gu, Suwon 443-721, Korea

Tel: +82-31-219-5284, Fax: +82-31-219-5209, E-mail: syyim@ajou.ac.kr

(c) This is an open-access article distributed under the terms of the Creative Commons Attribution Non-Commercial License (http://creativecommons. org/licenses/by-nc/4.0) which permits unrestricted noncommercial use, distribution, and reproduction in any medium, provided the original work is properly cited.

Copyright $\odot 2015$ by Korean Academy of Rehabilitation Medicine

\section{INTRODUCTION}

Congenital muscular torticollis (CMT) is one of the most frequent musculoskeletal disorders among children, with prevalence rates ranging from $0.3 \%$ to $3.92 \%$ $[1,2]$. CMT might be defined as a developmental disorder of the sternocleidomastoid muscle (SCM) that is characterized by fibrosis, eventually leading to shortening of the SCM and resulting in limited neck motion $[3,4]$.

About $90 \%$ of CMT cases can be cured with stretching exercises only, without musculoskeletal complications 
[1]. However, about $10 \%$ of CMT cases hardly respond to stretching exercises. Surgical release is needed for this severe subgroup of CMT to minimize the secondary musculoskeletal complications of CMT [3]. Typical musculoskeletal complications of CMT in terms of craniofacial asymmetries include depression of the frontal bone and zygomatic arch on the CMT side, posteriorly positioned ear on the CMT side, deformational plagiocephaly on the non-CMT side, and deviation of the chin toward the nonCMT side [5-7].

The timing of surgery for CMT is an important factor that influences outcome. The best surgical outcomes are obtained before 5 years of age. In older individuals, surgical efficacy is reduced owing to irreversible musculoskeletal deformity. Most subjects with neglected CMT complain of secondary musculoskeletal deformities, in addition to pain and deficit of the neck motion for the remainder of their lives [8].

Although some studies suggest that surgical release of the SCM in neglected CMT is effective for cosmetic purposes, pain reduction, and functional improvement, there can be drawbacks. Based on our literature review, no report has been published on the natural history of craniofacial asymmetry of subjects with neglected CMT as they age. If the craniofacial asymmetry in subjects with neglected CMT worsens even after growth cessation, the progression of craniofacial asymmetry needs to be decelerated through surgical release, even for adults with neglected CMT.

The objective of this study was to evaluate the relationship between severity of craniofacial asymmetry and age in adults with neglected CMT by quantitatively assessing craniofacial asymmetry using craniofacial three-dimensional computed tomography (3D-CT).

\section{MATERIALS AND METHODS}

This was a retrospective study conducted in a single tertiary medical center. This research was approved by the Institutional Review Board of Ajou Medical Center.

\section{Subjects}

Among the subjects who visited the Clinic for Torticollis for the symptoms of abnormal head and neck postures between January 2009 and December 2013, subjects diagnosed with CMT who aged 18 years old or older at the

Table 1. Anatomical landmarks used in cephalometric measurements

\begin{tabular}{|c|c|c|}
\hline Landmark & Abbr. & Description \\
\hline \multicolumn{3}{|l|}{ Superior cranial view } \\
\hline Orbitale superius & OS & $\begin{array}{l}\text { The most anterior point directly above the most superior point of the superior } \\
\text { orbital rim }\end{array}$ \\
\hline \multicolumn{3}{|l|}{ Frontal view } \\
\hline Glabella & G & The most anterior point of frontal bone between the supraorbital ridge \\
\hline Nasion & $\mathrm{N}$ & The intersection point of the internasal and frontonasal sutures \\
\hline Top of the nasal spine & TNS & The highest point on the superior aspect of the nasal septum \\
\hline Anterior nasal spine & ANS & The centre of the intersection of the nasal septum and the palate \\
\hline Mentum & M & The lowest point of the mandibular symphysis \\
\hline Latero-orbitale & LO & $\begin{array}{l}\text { The intersection of the temporal line of the frontal bone and the internal } \\
\text { orbital margin }\end{array}$ \\
\hline Zygonion & $\mathrm{ZY}$ & The most lateral aspect of the zygomatic arch \\
\hline Gonion & GO & The midpoint on the curvature at the angle of the mandible \\
\hline \multicolumn{3}{|l|}{ Inferior cranial view } \\
\hline Mastoid process & MA & The most anterior point of the mastoid process \\
\hline Zygomaxillare & $\mathrm{ZM}$ & The most anterior point of each zygomaticomaxillary sutures \\
\hline Palatine midpoint & $\mathrm{PM}$ & The deepest midpoint of the palatine bone \\
\hline Opisthion & $\mathrm{O}$ & The most posterior point on the posterior margin of the foramen magnum \\
\hline Occipital protuberance & $\mathrm{OP}$ & $\begin{array}{l}\text { Projection on the external surface of the squamous part of the occipital bone } \\
\text { in the midline }\end{array}$ \\
\hline
\end{tabular}


time of craniofacial 3D-CT scan were included. Exclusion criteria were as follows: 1 ) subjects with a previous history of surgery or injection of botulinum toxin into the SCM for CMT; 2) subjects who had congenital disease that might incur craniofacial asymmetry, such as craniovertebral junction anomaly, segmentation anomaly of the vertebra, Goldenhar syndrome, Treacher Collins syndrome, and CHARGE syndrome, etc.

A diagnosis of CMT was made for the subjects who showed both of the following conditions: 1) shortening of the unilateral SCM, ending up with ipsilateral lateral flexion of the head and/or rotation of the chin toward the contralateral shoulder and 2) multiple or large low signal intensities within the SCM in magnetic resonance imaging, as previous described [9].
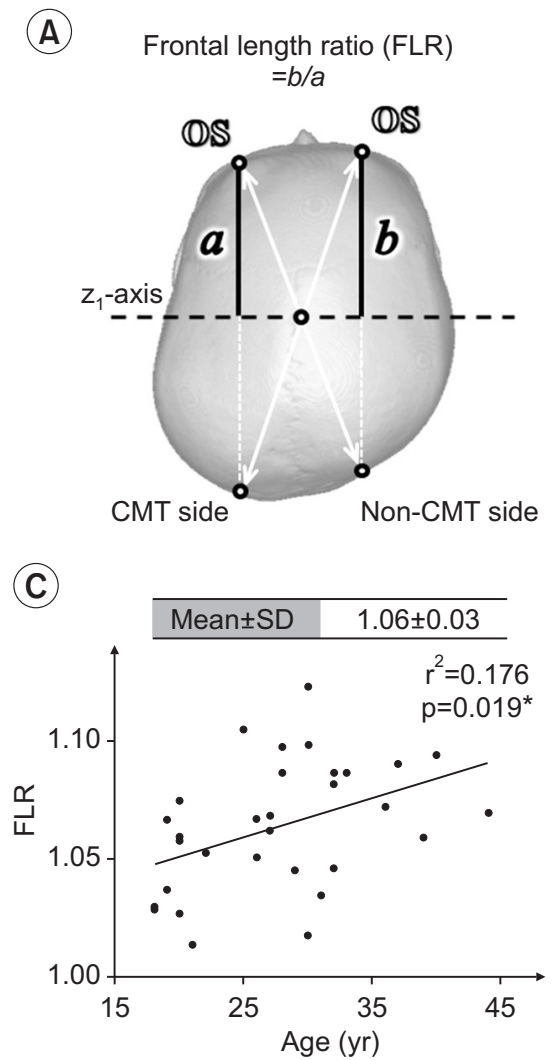

Craniofacial 3D-CT was performed when subjects showed grossly obvious craniofacial deformity due to neglected CMT. Craniofacial 3D-CT (scan parameters: 120 $\mathrm{kVp}, 400 \mathrm{mAs}$, scan time, $750 \mathrm{~ms}$; matrix size, 512×512) was performed with multi-detector row helical CT (Philips, Eindhoven, The Netherlands). The CT scans were reviewed with a PiViewSTAR program (Infinitt, Seoul, Korea).

Limitation in range of neck motion and radiographic cervicomandibular angle were used for determining the clinical severity of CMT. A retrospective chart review of range of neck motion was performed for all cases. Range of neck motion was measured by using a handheld goniometer and was compared with that of the contralateral side to calculate the motion deficit. The cervicoman-

(B)
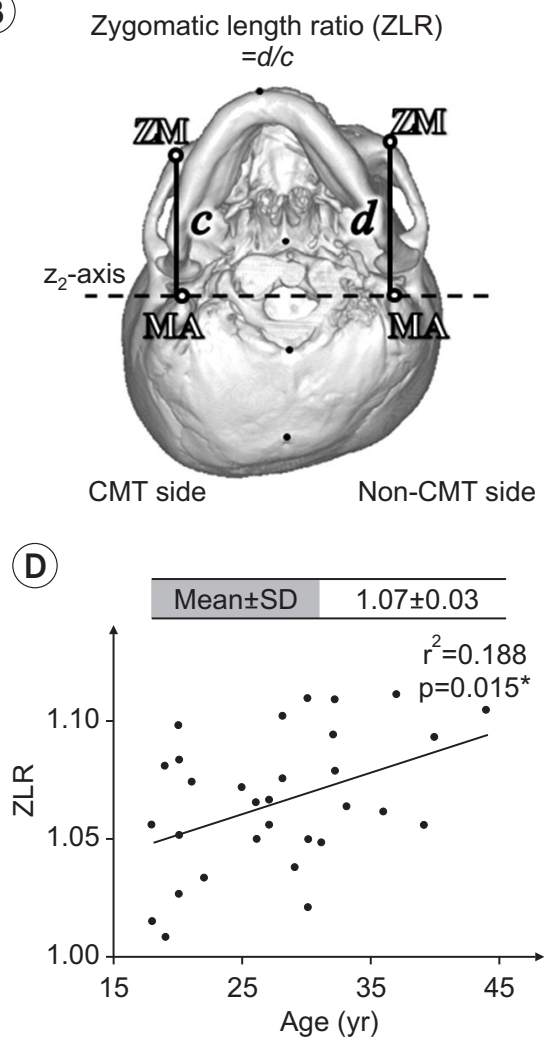

Fig. 1. Measurement of the anteroposterior length asymmetry on the axial plane. (A) The FLR, $b / a$, which represents the anteroposterior length asymmetry of the OS on the CMT side in comparison with that on the non-CMT side on the superior cranial view. The $z_{1}$-axis was defined as the coronal line perpendicular to the sagittal plane, which crosses the intersection of the 2 diagonals from the OS. (B) The ZLR, $d / c$, which represents the anteroposterior length asymmetry of the ZM on the CMT side in comparison with that on the non-CMT side on the inferior cranial view. The $Z_{2}$-axis was defined as the line connecting the bilateral MA. (C) Linear regression analysis between FLR and the subjects' ages. (D) Linear regression analysis between ZLR and the subjects' ages. The scatter plots of FLR and ZLR show statistically significant linear correlation $\left({ }^{*} \mathrm{p}<0.05\right)$ with age. OS, orbitale superius; CMT, congenital muscular torticollis; ZM, zygomaxillare; MA, mastoid processes. 
dibular angle, defined as the angle between a line along the upper border of the $\mathrm{C} 7$ vertebral body and a line connecting the lower margins of the mandibular angle, was measured on the anteroposterior radiograph of the cervical spine to quantify the degree of head tilt [10].

\section{Quantitative analysis of asymmetry on craniofacial 3D-} CT

Anatomical landmarks were identified on one of three standard 3D-CT views (superior cranial, inferior cranial, and frontal views), which were vertical, caudal, and frontal surface projection images, respectively, based on the Frankfort horizontal plane $[7,11,12]$. All the anatomical landmarks used in this study are described in Table 1. Quantitative measurements of asymmetry on craniofa- cial 3D-CT were independently and blindly performed by two of the first three authors. After inter-rater reliability was tested for quantitative measurement of craniofacial $3 \mathrm{D}$-CT findings, the mean values measured by the two authors was used for linear regression analysis between craniofacial asymmetry and subject age.

\section{Severity of cranial asymmetry}

The cranial vault asymmetry index (CVAI) was used to measure the severity of plagiocephaly $[13,14]$. The diagonal diameters were obtained from the orbitale superius (OS) directly above the most superior point of the superior orbital rim to the occipital landmark, which is defined by a parallel line directed posteriorly from the OS on the opposite side (Fig. 1A). CVAI (\%) was calculated as [(lon-

(A)
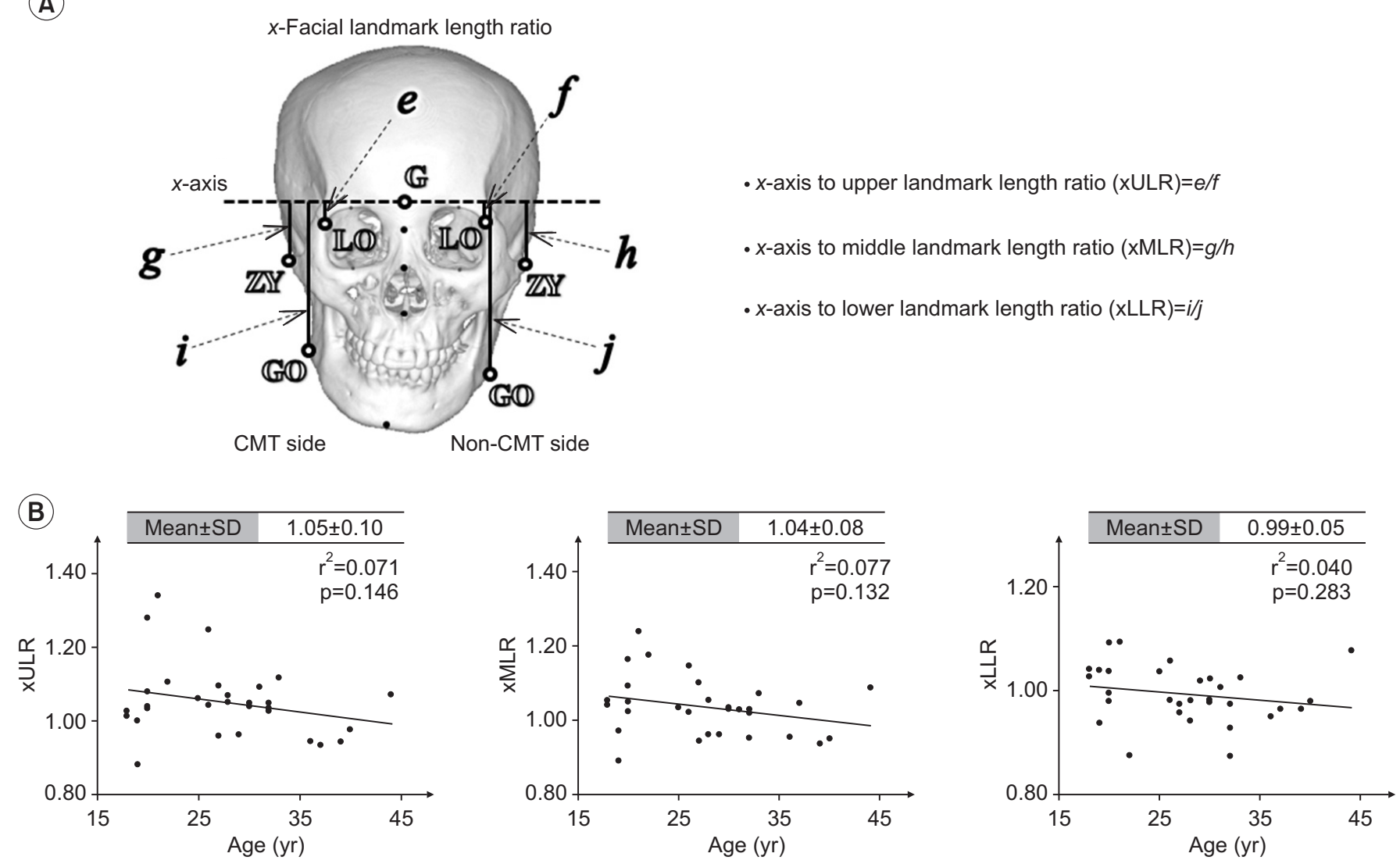

Fig. 2. Measurement of the vertical length asymmetry on the coronal plane. (A) A mid-sagittal line was constructed by joining the landmarks, namely the glabella ' $G$ ', nasion ' $N$ ', and top of the nasal spine 'TNS', to form the $y_{1}$-axis and perpendicular line with origins at $G$, defined as the $x$-axis on the frontal view. The xULR, $e / f$, represents the vertical length asymmetry of the LO from the $x$-axis on the CMT side in comparison with that on the non-CMT side. The xMLR, $g / h$, represents the vertical length asymmetry of the ZY. The xLLR, $i / j$, represents the vertical length asymmetry of the GO on the CMT side in comparison with that on the non-CMT side. (B) Linear regression analyses between xULR, xMLR, xLLR, and the subjects' ages. LO, latero-orbitale; CMT, congenital muscular torticollis; ZY, zygonion; GO, gonion. 
ger diagonal shorter diagonal) / shorter cranial diagonal] $\times 100$. Cranial asymmetry was considered significant if CVAI $>3.5 \%$, and was classified as mild (3.5\%-7\%), moderate $(7 \%-12 \%)$, or severe $(>12 \%)$.

\section{Measurement of anteroposterior length asymmetry on} the axial plane

Anteroposterior length asymmetry of the OS and zygomaxillare (ZM) was measured as shown in Fig. 1A and B. The frontal length ratio (FLR), $b / a$, represents the length asymmetry of the OS on the CMT side in comparison with that on the non-CMT side in the anteroposterior direction of the axial plane, in the superior cranial view. The $z_{1}$-axis was defined as the coronal line perpendicular to the sagittal plane, which crosses the intersection of the
2 previously mentioned diagonals. The zygomatic length ratio (ZLR), $d / c$, represents the anteroposterior length asymmetry of the ZM on the CMT side in comparison with that on the non-CMT side, in the inferior cranial view. The $z_{2}$-axis was defined as the line connecting the bilateral anterior margin of the mastoid processes (MA), and $\mathrm{ZM}$ is the most anterior point of each zygomaticomaxillary sutures.

\section{Measurement of vertical length asymmetry on the coronal plane}

The vertical length asymmetry of the anterior facial landmarks was measured as shown in Fig. 2A. A midsagittal line was constructed by joining the landmarks, namely the glabella (G), nasion (N), and top of the nasal

(A)

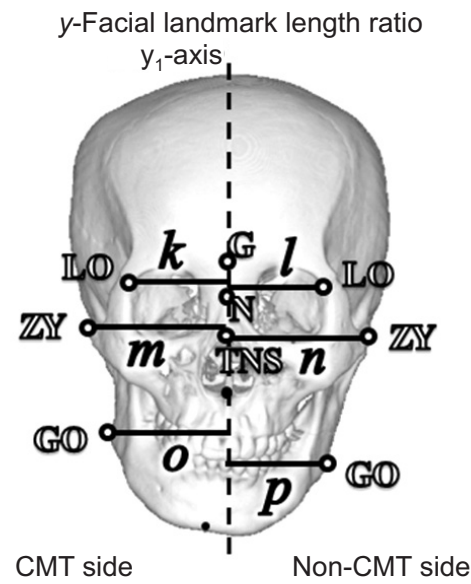

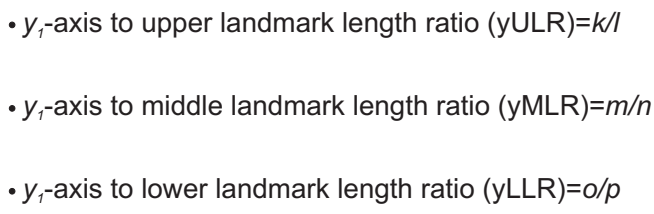

- $y_{1}$-axis to lower landmark length ratio $(y L L R)=0 / p$
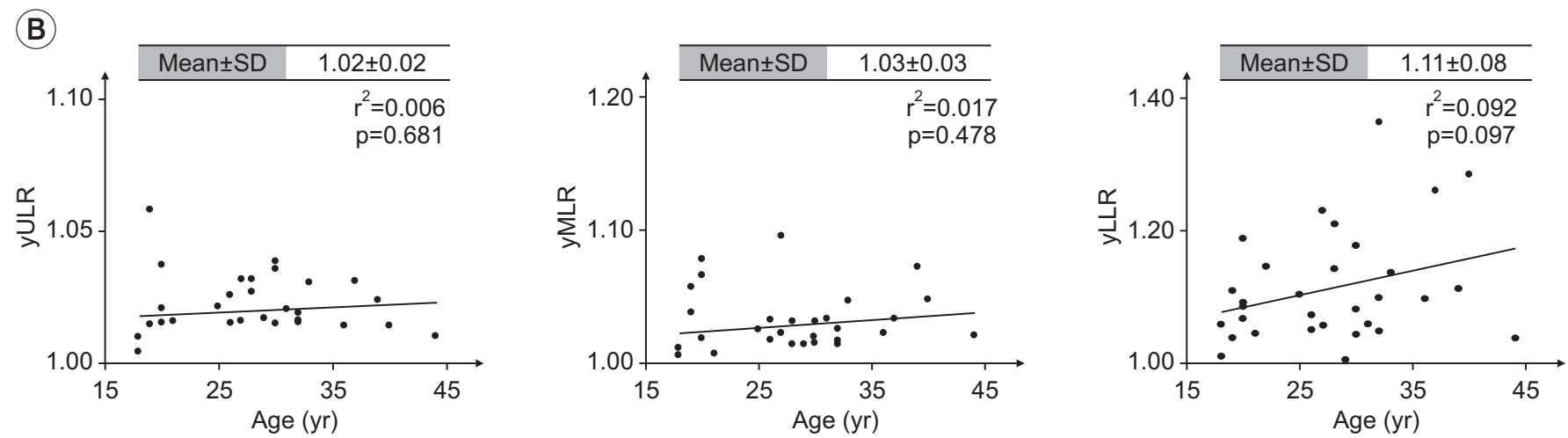

Fig. 3. Measurement of the lateral length asymmetry on the coronal plane. (A) The distances from the $y_{1}$-axis to the paired landmarks mentioned in Fig. 2A were measured in frontal view. The yULR, $k / l$, which represents the lateral length asymmetry of the LO from the $y_{1}$-axis on the CMT side in comparison with that on the non-CMT side. The yMLR, $m / n$, represents the lateral length asymmetry of ZY. The yLLR, $o / p$, represents the lateral length asymmetry of GO on the CMT side in comparison with that on the non-CMT side. (B) Linear regression analyses between yULR, yMLR, yLLR, and the subjects' ages. LO, latero-orbitale; CMT, congenital muscular torticollis; ZY, zygonion; GO, gonion. 
spine (TNS) to form the $y_{1}$-axis, and the perpendicular line with origins at the $\mathrm{G}$ was defined as the $x$-axis in the frontal view. The $x$-upper landmark length ratio (xULR), e/f, represents the vertical length asymmetry of the latero-orbitale (LO), the intersection of the temporal line of the frontal bone and internal orbital margin from the $x$-axis on the CMT side in comparison with that on the non-CMT side. The $x$-middle landmark length ratio (xMLR), $g / h$, represents the vertical length asymmetry of the zygonion (ZY) on the CMT side in comparison with that on the non-CMT side. The $x$-lower landmark length ratio (xLLR), $i / j$, represents the vertical length asymmetry of the gonion (GO) on the CMT side in comparison with that on the non-CMT side. $\mathrm{ZY}$ is the most lateral point on the outline of each zygomatic arch, and GO is the mid- point on the curvature at the angle of the mandible.

Measurement of lateral length asymmetry on the coronal plane

Lateral length asymmetry of facial landmarks LO, ZY, and GO was measured as depicted in Fig. 3A. The $y$ upper landmark length ratio (yULR), $k / l$, represents the lateral length asymmetry of the LO from the $y_{1}$-axis on the CMT side in comparison with that on the non-CMT side. The $y$-middle landmark length ratio (yMLR), $m / n$, represents the lateral length asymmetry of the $\mathrm{ZY}$ on the CMT side in comparison with that on the non-CMT side. The $y$-lower landmark length ratio (yLLR), $o / p$, represents the lateral length asymmetry of the GO on the CMT side in comparison with that on the non-CMT side.

(A)
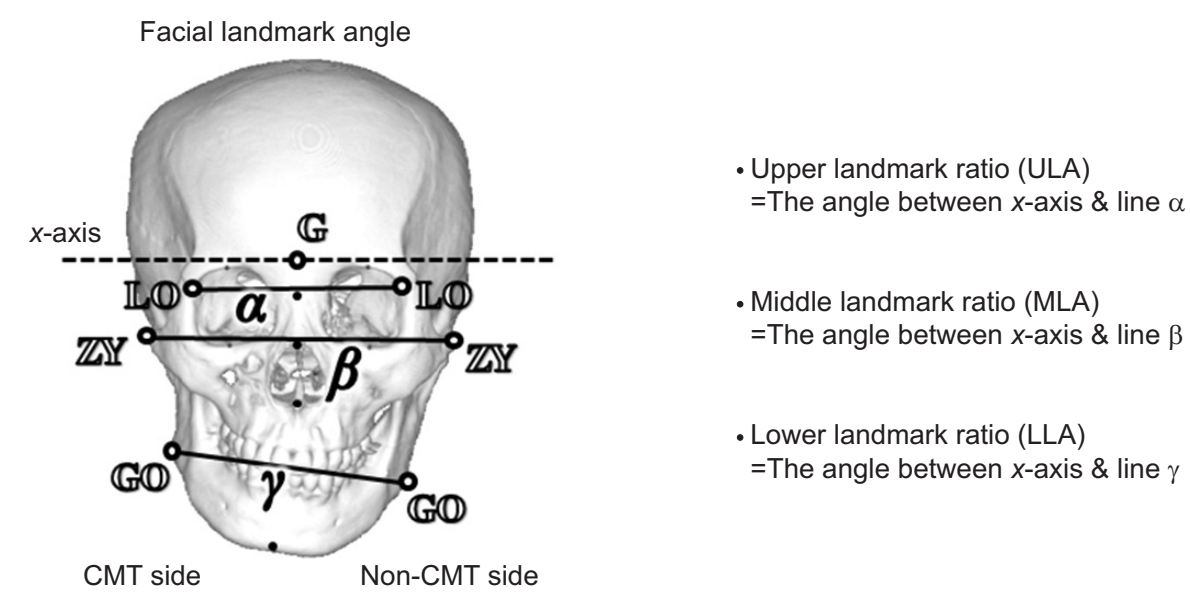

(B)
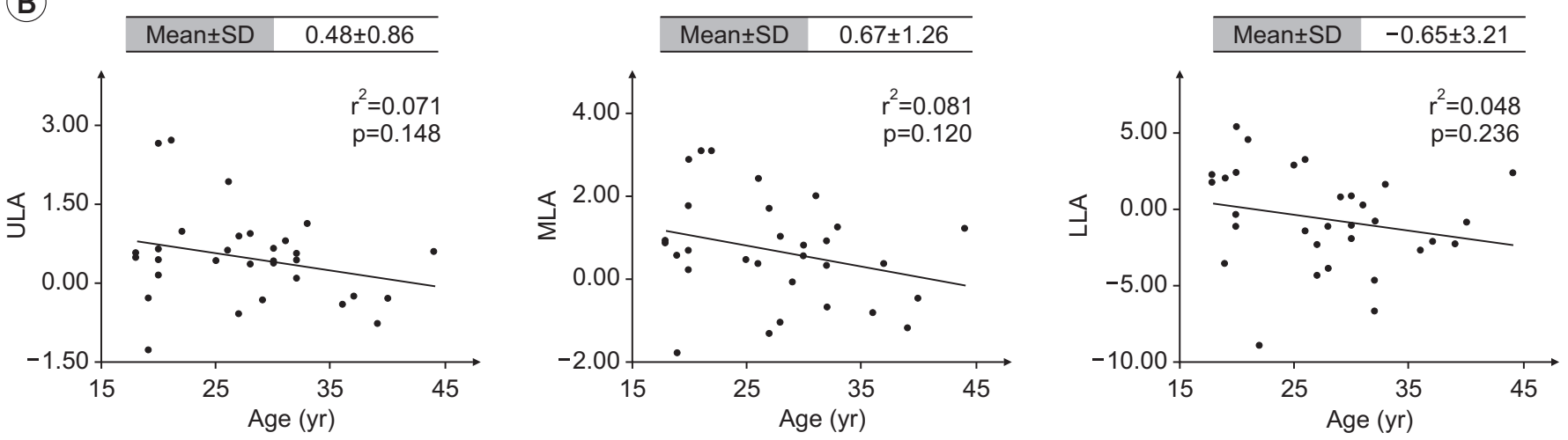

Fig. 4. Measurement of the vertical displacement on the coronal plane. (A) The ULA, which represents the vertical displacement of the LO on the CMT side in comparison with that on the non-CMT side, is the angle between the $x$-axis and line $\alpha$. The MLA, which represents the vertical displacement of the ZY on the CMT side in comparison with that on the non-CMT side, is the angle between the $x$-axis and line $\beta$. The ULA, which represents the vertical displacement of the GO on the CMT side in comparison with that on the non-CMT side, is the angle between the $x$-axis and line $\gamma$. (B) Linear regression analyses between ULA, MLA, LLA, and the subjects' ages. LO, latero-orbitale; CMT, congenital muscular torticollis; ZY, zygonion; GO, gonion. 
Measurement of vertical displacement on the coronal plane

Vertical displacement of facial landmarks LO, ZY, and GO was measured as shown in Fig. 4A. The upper landmark angle (ULA), which represents the vertical displacement of the LO on the CMT side in comparison with that on the non-CMT side, is the angle between the $x$-axis and line $\alpha$. The middle landmark angle (MLA), which represents the vertical displacement of the $\mathrm{ZY}$ on the CMT side in comparison with that on the non-CMT side, is the angle between the $x$-axis and line $\beta$. The lower landmark angle (ULA), which represents the vertical displacement of the GO on the CMT side in comparison with that on the non-CMT side, is the angle between the $x$-axis and line $\gamma$.

Measurement of mandibular rotation on the coronal and axial planes

To measure the degree of mandibular rotation, the frontal axis deviation (FAD) and caudal axis deviation (CAD) were measured as shown in Fig. $5 \mathrm{~A}$ and B. FAD is the angle formed by the intersection of the $y_{1}$-axis and line $\delta$, which represents the frontal mandibular axis defined by a line connecting the anterior nasal spine (ANS) and mentum (M) in the frontal view. CAD is the angle between the $y_{2}$-axis and line $\varepsilon$, which represents the caudal mandibular axis. The $y_{2}$-axis was defined by joining the landmarks occipital protuberance (OP), opisthion (O). The caudal mandibular axis was constructed by joining the landmarks $\mathrm{M}$, and palatine midpoint (PM) in the inferior cranial view.

\section{Statistical analysis}

Statistical Package for the Social Sciences (SPSS) ver. 19.0 (IBM, Armonk, NY, USA) was used for the statistical analysis. The intra-class correlation coefficients (ICC) between the two investigators were calculated for inter-rater reliability. ICC scores $>0.70$ were considered reliable. Linear regression analysis was performed to evaluate the relationship between the cephalometric parameters and age of the subjects. If the relationship between cephalometric parameters and subjects' age was statistically significant in the simple linear regression analysis, a multiple regression analysis was performed, with covari-
A Frontal axis deviation (FAD) $=$ The angle between $\mathrm{y}_{1}$-axis and line $\delta$

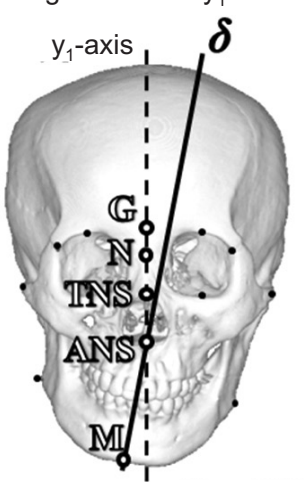

CMT side

Non-CMT side

(C)

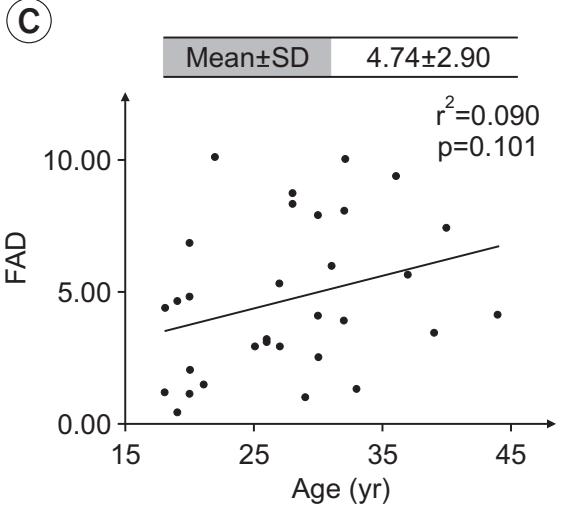

(B)

Caudal axis deviation (CAD) $=$ The angle between $\mathrm{y}_{2}$-axis and line $\varepsilon$

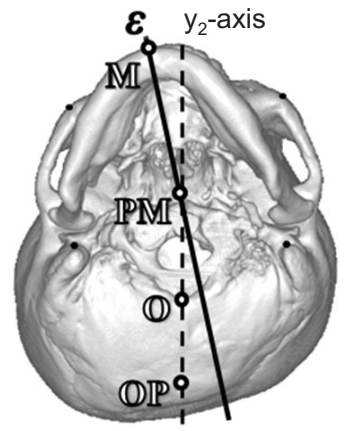

CMT side

Non-CMT side

(D)

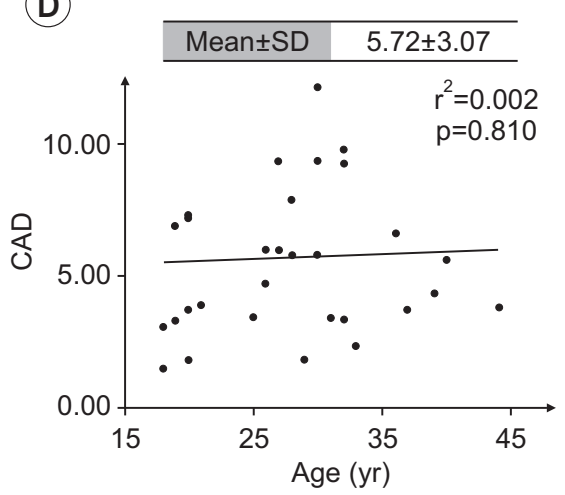

Fig. 5. Measurement of the mandibular axis rotation. (A) The FAD is the angle formed by the intersection of the $y_{1}$-axis and line $\delta$, which represents the frontal mandibular axis, defined by a line connecting the nasal spine (ANS) and $\mathrm{M}$ on the frontal view. (B) The CAD is the angle between the $y_{2}-$ axis and line $\varepsilon$, which represents the caudal mandibular axis. The $y_{2}$-axis was defined by joining the landmarks $\mathrm{OP}$ and $\mathrm{O}$. The caudal mandibular axis was constructed by joining the landmarks M, PM in the inferior cranial view. (C) Linear regression analysis between FAD and the subjects' ages. (D) Linear regression analysis between CAD and the subjects' ages. ANS, anterior nasal spine; TNS, top of the nasal spine; $\mathrm{M}$, mentum; OP, occipital protuberance; $\mathrm{O}$, opisthion; $\mathrm{PM}$, palatine midpoint; G, glabella; N, nasion. 
ates including limitation in range of neck motion or cervicomandibular angle. Statistical significance was set at $\mathrm{p}<0.05$.

\section{RESULTS}

\section{Characteristics of the subjects}

Thirty-one subjects ( 9 men and 22 women) who met the inclusion criteria were included in this retrospective study. The characteristics of the subjects are presented in Table 2. Age at the time of 3D-CT was $27.71 \pm 7.02$ years (range, 18-44 years). The involved CMT side was the right in 21 subjects (67.7\%) and left in 10 subjects (32.3\%).

Quantitative analysis on craniofacial 3D-CT with interrater reliability

The results of the quantitative analysis of asymmetry on craniofacial 3D-CT as measured by the two investigators are presented in Table 3 along with the ICC values. All the ICC values were higher than 0.70 , suggesting a good inter-rater reliability of all the measurements $(p<0.05)$.

\section{Severity of cranial asymmetry}

Based on CVAI, 26 subjects (83.9\%) had a significant cranial asymmetry, which include 8 mild cases, 17 moderate cases, and 1 severe case.

\section{Anteroposterior length asymmetry on the axial plane}

FLR (i.e., the anteroposterior length asymmetry of the frontal bone on the axial plane) was $1.06 \pm 0.03$, which increased significantly with ages in the linear regression analysis $\left(\mathrm{r}^{2}=0.176, \mathrm{p}=0.019\right)$ (Fig. 1C). The relationship between the FLR and age was still statistically significant after adjusting for limitation in range of neck flexion and lateral flexion (adjusted $\mathrm{r}^{2}=0.141, \mathrm{p}=0.045$ ) or cervicomandibular angle (adjusted $\mathrm{r}^{2}=0.231, \mathrm{p}=0.010$ ). ZLR (i.e., the anteroposterior length asymmetry of the zygomatic arch on the axial plane) was $1.07 \pm 0.03$, which also increased significantly along with age in the linear regression analysis $\left(r^{2}=0.188, p=0.015\right)$ (Fig. 1D). The relationship between the ZLR and age was still statistically significant after adjusting for limitation in range of neck flexion and lateral flexion (adjusted $\mathrm{r}^{2}=0.227, \mathrm{p}=0.019$ ) or cervicomandibular angle (adjusted $\mathrm{r}^{2}=0.426, \mathrm{p}<0.001$ ).

\section{Vertical length asymmetry on the coronal plane}

xULR, xMLR, and xLLR (i.e., the vertical length asymmetry of the anterior facial landmarks on the coronal plane was $1.04 \pm 0.09,1.03 \pm 0.08$, and $0.99 \pm 0.05$, respectively. None of these was statistically significant with age in linear regression analysis $\left(\mathrm{r}^{2}=0.071,0.077\right.$, and 0.040 , respectively; all $\mathrm{p}>0.05$ ) (Fig. $2 \mathrm{~B}$ ).

\section{Lateral length asymmetry on the coronal plane}

yULR, yMLR, and yLLR (i.e., the lateral length asymmetry of the same paired landmarks on the coronal plane) was $1.02 \pm 0.02,1.03 \pm 0.03$, and $1.12 \pm 0.08$, respectively. None of these was statistically significant with age in linear regression analysis $\left(\mathrm{r}^{2}=0.006,0.017\right.$, and 0.092 , respectively; all p>0.05) (Fig. 3B).

\section{Vertical displacement on the coronal plane}

ULA, MLA, and LLA (i.e., the vertical displacement of the anterior facial landmarks on the coronal plane was $0.48 \pm 0.86,0.67 \pm 1.26$, and $-0.65 \pm 3.21$, respectively. None of these was statistically significant with age in linear regression analysis $\left(\mathrm{r}^{2}=0.071,0.081\right.$, and 0.048 , respectively; all $\mathrm{p}>0.05$ ) (Fig. 4B).

Table 2. Characteristics of the 31 subjects

\begin{tabular}{lc}
\hline \multicolumn{1}{c}{ Characteristic } & Value \\
\hline Age at the time of 3D-CT scan (yr) & $27.71 \pm 7.02(18-44)$ \\
\hline Gender (male:female) & $9: 22$ \\
Affected side of congenital muscular torticollis (right:left) & $21: 10$ \\
\hline Limitation in range of neck rotation ( $\left.{ }^{\circ}\right)$ & $20.65 \pm 8.14$ \\
Limitation in range of lateral neck flexion (o) & $32.58 \pm 15.80$ \\
Cervicomandibular angle $\left(^{\circ}\right)$ & $13.38 \pm 6.81$ \\
\hline
\end{tabular}

Values are presented as mean \pm standard deviation (range) or number.

3D-CT, three-dimensional computed tomography. 
Table 3. Inter-rater reliability of the cephalometric measurements

\begin{tabular}{|c|c|c|c|c|c|}
\hline Cephalometric analysis & Diagram & Investigator $^{a)}$ & Mean \pm SD & ICC & p-value \\
\hline \multicolumn{6}{|c|}{ Anteroposterior length asymmetry in axial plane } \\
\hline \multirow[t]{2}{*}{ Frontal length ratio } & Fig. 1A & 1 & $1.07 \pm 0.03$ & 0.91 & $0.01^{*}$ \\
\hline & & 2 & $1.06 \pm 0.03$ & & \\
\hline \multirow[t]{2}{*}{ Zygomatic length ratio } & Fig. 1B & 1 & $1.07 \pm 0.03$ & 0.90 & $0.01^{*}$ \\
\hline & & 2 & $1.06 \pm 0.03$ & & \\
\hline \multicolumn{6}{|c|}{ Vertical length asymmetry in coronal plane } \\
\hline \multirow[t]{2}{*}{$\mathrm{x}$-Upper landmark length ratio } & Fig. 2A & 1 & $1.04 \pm 0.09$ & 0.85 & $0.04^{*}$ \\
\hline & & 3 & $1.06 \pm 0.10$ & & \\
\hline \multirow[t]{2}{*}{$\mathrm{x}$-Middle landmark length ratio } & Fig. 2A & 1 & $1.03 \pm 0.08$ & 0.91 & $0.03^{*}$ \\
\hline & & 3 & $1.04 \pm 0.08$ & & \\
\hline \multirow[t]{2}{*}{$\mathrm{x}$-Lower landmark length ratio } & Fig. 2A & 1 & $0.99 \pm 0.05$ & 0.97 & $0.04^{*}$ \\
\hline & & 3 & $1.00 \pm 0.06$ & & \\
\hline \multicolumn{6}{|c|}{ Lateral length asymmetry in coronal plane } \\
\hline \multirow[t]{2}{*}{$\mathrm{y}$-Upper landmark length ratio } & Fig. 3A & 1 & $1.02 \pm 0.02$ & 0.71 & $0.03^{*}$ \\
\hline & & 3 & $1.02 \pm 0.02$ & & \\
\hline \multirow[t]{2}{*}{ y-Middle landmark length ratio } & Fig. 3A & 1 & $1.03 \pm 0.03$ & 0.89 & $0.03^{*}$ \\
\hline & & 3 & $1.03 \pm 0.03$ & & \\
\hline \multirow[t]{2}{*}{$\mathrm{y}$-Lower landmark length ratio } & Fig. 3A & 1 & $1.12 \pm 0.08$ & 0.93 & $0.04^{*}$ \\
\hline & & 3 & $1.11 \pm 0.09$ & & \\
\hline \multicolumn{6}{|l|}{ Vertical displacement in coronal plane } \\
\hline \multirow[t]{2}{*}{ Upper landmark angle (o) } & Fig. $4 \mathrm{~A}$ & 1 & $0.41 \pm 0.92$ & 0.86 & $<0.001^{* * *}$ \\
\hline & & 3 & $0.55 \pm 0.86$ & & \\
\hline \multirow[t]{2}{*}{ Middle landmark angle (o) } & Fig. $4 \mathrm{~A}$ & 1 & $0.70 \pm 1.26$ & 0.89 & $<0.001^{* * *}$ \\
\hline & & 3 & $0.64 \pm 1.32$ & & \\
\hline \multirow[t]{2}{*}{ Lower landmark angle $\left({ }^{\circ}\right)$} & Fig. $4 \mathrm{~A}$ & 1 & $-0.70 \pm 3.13$ & 0.99 & $<0.001^{* * *}$ \\
\hline & & 3 & $-0.61 \pm 3.31$ & & \\
\hline \multicolumn{6}{|l|}{ Mandibular rotation in coronal plane } \\
\hline \multirow[t]{2}{*}{ Frontal axis deviation ( ${ }^{\circ}$ ) } & Fig. 5A & 1 & $4.82 \pm 2.91$ & 0.97 & $<0.001^{* * *}$ \\
\hline & & 3 & $4.66 \pm 2.94$ & & \\
\hline \multicolumn{6}{|l|}{ Mandibular rotation in axial plane } \\
\hline \multirow[t]{2}{*}{ Caudal axis deviation $(\mathrm{o})$} & Fig. 5B & 1 & $5.58 \pm 3.18$ & 0.97 & $<0.001^{* * *}$ \\
\hline & & 2 & $5.85 \pm 3.02$ & & \\
\hline
\end{tabular}

ICC, intra-class correlation coefficient.

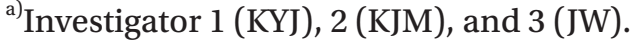

${ }^{*} \mathrm{p}<0.05,{ }^{* *} \mathrm{p}<0.01,{ }^{* * *} \mathrm{p}<0.001$.

Mandibular rotation on the coronal and axial planes

FAD and CAD, which represent the deviation of the mandibular axis on the coronal and axial planes, were $4.82^{\circ} \pm 2.91^{\circ}$ and $5.58^{\circ} \pm 3.18^{\circ}$, respectively. Both were not statistically significant with age in linear regression analysis $\left(\mathrm{r}^{2}=0.090, \mathrm{p}=0.101\right.$ and $\mathrm{r}^{2}=0.002, \mathrm{p}=0.810$, respectively) (Fig. 5C and D).

\section{DISCUSSION}

To our knowledge, this study is the first to quantitatively evaluate craniofacial abnormalities, focusing on CMT patients aged 18 years or older. The aim was to evaluate the relationship between age and the severity of craniofacial asymmetry in patients with neglected CMT. 
Craniofacial 3D-CT allows objective and quantitative assessments of the level of craniofacial asymmetry. Facial asymmetry is progressive if the contracted SCM is not released, although cranial asymmetry was already determined in patients younger than 6 months old [5]. However, the authors reported that the intercommissural angle was only measured by frontal photo analysis to represent facial asymmetry. Another study reported a correlation of the severity of craniofacial deformity with age, as analyzed using craniofacial 3D-CT images obtained from 14 CMT patients aged 1 month to 24 years [7]. However, most of the subjects were infants and juveniles, with only three adults.

The present study found a correlation of the severity of craniofacial asymmetry with age in terms of anteroposterior length asymmetry of the ipsilateral frontal bone and zygomatic arch on the axial plane in adult CMT patients. This may have reflected the ongoing shortening of the SCM on the affected side, which remains even after growth cessation. Unresolved traction force of the affected SCM and the contracted neighboring soft tissue may cause continuous mild torsion of craniofacial structures.

Inferiorly and laterally shifted facial landmarks on the CMT side on the coronal plane were noted (except vertical displacement of the GO), as well as a rotated mandibular axis toward the affected side on the coronal and axial planes. Unlike anteroposterior length asymmetry, none of these was considered progressive in the linear regression analysis with age.

We cannot fully explain this particular result on the axial plane, but it was observed that readings of the facial landmark length ratio and frontal mandibular axis deviation influenced the other readings. In case of, for example, right CMT, the GO level on the right side becomes raised with a considerable mandibular rotation toward the right side, which affects the estimation of the $x$-axis to the lower landmark length ratio (significant negative correlation was identified in the correlation analysis between the xLLR and FAD; $r=-0.619$ and $p<0.001$ ). In addition, there are possibilities of displacement underestimation of bilateral landmarks on the axial plane in the frontal view.

Although frontal photograph and simple radiographic analyses are performed to assess asymmetry in adult patients with CMT, in many cases they may not be enough to evaluate an anteroposterior length asymmetry, as shown in this study, which implies that 3D-CT may be beneficial.

There are pros and cons regarding treatments, surgical corrections inter alia, for adult patients who have neglected or unresolved CMT [15]. Many have refused such surgeries, taking into consideration the high rate of complications, marginal treatment effectiveness, and ongoing abnormalities. It is recognized that not all surgeries may result in the correction of craniofacial abnormalities, particularly for patients aged 5 years or older [16-18]. Nonetheless, as recently reported, interest is increasing on those methods in view of the fact that any surgical correction may improve cosmetic and functional aspects along with the alleviation of pain related to it [8]. Others also reported improvement of cosmesis and range of neck movement after unipolar resection of the affected SCM [19].

According to the present study, surgical release of the affected SCM in neglected CMT patients may be required to prevent further exacerbation of craniofacial asymmetry, when the severity increases with age throughout the patient's adult life.

The strength of this study lies on the quantitative and objective analyses conducted based on 3D-CT data from the largest adult population with neglected CMT among studies regarding craniofacial asymmetry in CMT patients. The inter-rater reliability of the measurements between the two investigators was verified through an ICC test.

Limitation of this study is its retrospective nature, which may inherently contain the possibility of selection bias. Although this study had the largest targeted adult population among studies regarding craniofacial asymmetry in CMT patients, a longitudinal study targeting more subjects may lead to more precise results.

In conclusion, craniofacial asymmetry in terms of anteroposterior length asymmetry of the frontal bone and zygomatic arch on the axial plane became more severe with age even in the adults, based on the quantitative evaluation of 3D-CT images from 31 neglected CMT subjects aged 18 years or older. Assessment of anteroposterior length asymmetry on the axial plane seems to be the most accurate tool for measuring craniofacial asymmetry in adult CMT subjects. This finding may enhance our understanding of therapeutic strategies, such as careful monitoring and surgical release, to prevent progression 
of craniofacial asymmetry in adults with neglected CMT.

\section{CONFLICT OF INTEREST}

No potential conflict of interest relevant to this article was reported.

\section{REFERENCES}

1. Cheng JC, Wong MW, Tang SP, Chen TM, Shum SL, Wong EM. Clinical determinants of the outcome of manual stretching in the treatment of congenital muscular torticollis in infants: a prospective study of eight hundred and twenty-one cases. J Bone Joint Surg Am 2001;83A:679-87.

2. Chen MM, Chang HC, Hsieh CF, Yen MF, Chen TH. Predictive model for congenital muscular torticollis: analysis of 1021 infants with sonography. Arch Phys Med Rehabil 2005;86:2199-203.

3. Cheng JC, Tang SP, Chen TM, Wong MW, Wong EM. The clinical presentation and outcome of treatment of congenital muscular torticollis in infants: a study of 1,086 cases. J Pediatr Surg 2000;35:1091-6.

4. Yim SY, Yoon D, Park MC, Lee IJ, Kim JH, Lee MA, et al. Integrative analysis of congenital muscular torticollis: from gene expression to clinical significance. BMC Med Genomics 2013;6 Suppl 2:S10.

5. Seo SJ, Yim SY, Lee IJ, Han DH, Kim CS, Lim H, et al. Is craniofacial asymmetry progressive in untreated congenital muscular torticollis? Plast Reconstr Surg 2013;132:407-13.

6. Lee DY, Song BW, Cho TJ, Choi IH, Chung CY, Yoo WJ. Craniofacial Asymmetry in Congenital Muscular Torticollis Patients: A Study using Cephalometry. J Korean Orthop Assoc 2007;42:24-31

7. Yu CC, Wong FH, Lo LJ, Chen YR. Craniofacial deformity in patients with uncorrected congenital muscular torticollis: an assessment from three-dimensional computed tomography imaging. Plast Reconstr Surg 2004;113:24-33.

8. Lim KS, Shim JS, Lee YS. Is sternocleidomastoid muscle release effective in adults with neglected congenital muscular torticollis? Clin Orthop Relat Res 2014;472:1271-8.

9. Hwang JH, Lee HB, Kim JH, Park MC, Kwack KS, Han JD, Yim SY. Magnetic resonance imaging as a determinant for surgical release of congenital muscular torticollis: correlation with the histopathologic findings. Ann Rehabil Med 2012;36:320-7.

10. Shim JS, Jang HP. Operative treatment of congenital torticollis. J Bone Joint Surg Br 2008;90:934-9.

11. Chate RA. Facial scoliosis due to sternocleidomastoid torticollis: a cephalometric analysis. Int J Oral Maxillofac Surg 2004;33:338-43.

12. Lee JK, Moon HJ, Park MS, Yoo WJ, Choi IH, Cho TJ. Change of craniofacial deformity after sternocleidomastoid muscle release in pediatric patients with congenital muscular torticollis. J Bone Joint Surg Am 2012;94:e93.

13. Wilbrand JF, Wilbrand M, Malik CY, Howaldt HP, Streckbein P, Schaaf H, et al. Complications in helmet therapy. J Craniomaxillofac Surg 2012;40:341-6.

14. Loveday BP, de Chalain TB. Active counterpositioning or orthotic device to treat positional plagiocephaly? J Craniofac Surg 2001;12:308-13.

15. Khoury J. CORR Insights: Is sternocleidomastoid muscle release effective in adults with neglected congenital muscular torticollis? Clin Orthop Relat Res 2014;472:1279-80.

16. Tse P, Cheng J, Chow Y, Leung PC. Surgery for neglected congenital torticollis. Acta Orthop Scand 1987;58:270-2.

17. Oh I, Nowacek CJ. Surgical release of congenital torticollis in adults. Clin Orthop Relat Res 1978;(131):1415.

18. Ling CM. The influence of age on the results of open sternomastoid tenotomy in muscular torticollis. Clin Orthop Relat Res 1976;(116):142-8.

19. Chang SH, Ohtori S, Okawa A, Kawamura K, Saiki H, Nakada I, et al. A surgical treatment for adult muscular torticollis. Case Rep Orthop 2013;2013:965693. 